\title{
Quantitative 3D Information of Supported Pd/CMK-3 Catalysts at the Nanoscale
}

Wu Wang ${ }^{1,5}$, Di Wang ${ }^{1,2}$, Christian Kübel $^{1,2,3}$ and Alberto Villa ${ }^{4}$

1. Institute of Nanotechnology, Karlsruhe Institute of Technology, Eggenstein-Leopoldshafen, Germany.

2. Karlsruhe Nano Micro Facility, Karlsruhe Institute of Technology, Eggenstein-Leopoldshafen, Germany.

${ }^{3 .}$ Helmholtz-Institute Ulm for Electrochemical Energy Storage, Karlsruhe Institute of Technology, Ulm, Germany.

4. Dipartimento di Chimica, Università di Milano, Milano, Italy.

5. Joint Research Laboratory Nanomaterials, Technische Universität Darmstadt, Darmstadt, Germany.

Supported metal nanoparticles play an important role in areas such as energy storage/conversion and catalysis for sustainable production of fuels and chemicals [1]. Metal nanoparticles dispersed on porous carriers are widely used due to their large surface area and the confining effect, which can be used to tailor the desired catalytic properties. In heterogeneous catalysis, particle size, shape, location, dispersion, and accessibility of the active sites within the porous support have significant impact on the catalytic properties [2]. Quantitative three-dimensional (3D) information at the nanoscale is indispensable to understand the catalytic behaviors of supported nanoparticles. Electron tomography provides this $3 \mathrm{D}$ information to progress from flat $2 \mathrm{D}$ model substrates to more realistic $3 \mathrm{D}$ support morphologies where catalyst properties can be quantified after suitable image segmentation.

In this work, Pd nanoparticles were loaded on ordered porous CMK-3 carbon support (pore diameter 3-4 $\mathrm{nm})$ by three different methods: incipient wetness ( $\left.\mathrm{Pd}_{\mathrm{IW}} / \mathrm{CMK}-3\right)$, wet impregnation $\left(\mathrm{Pd}_{\mathrm{IMP}} / \mathrm{CMK}-3\right)$ and immobilization of preformed PVA stabilized nanoparticles ( $\left.\mathrm{Pd}_{\mathrm{PVA}} / \mathrm{CMK}-3\right)$. The three as-prepared $\mathrm{Pd} / \mathrm{CMK}-3$ catalysts were tested for the reduction of furfural. Several HAADF-STEM tomograms of the three Pd/CMK-3 catalysts before and after the catalytic reaction are reconstructed from tilt series with a tilt angle range of $\pm 70^{\circ}$ or higher. The size and location of the $\mathrm{Pd}$ nanoparticles are quantitatively measured after segmentation using Amira 6.1 and compared for the different types of loading.

The conversion after 3 hours of reaction time with the Pd $\mathrm{IW}_{\mathrm{IW}} / \mathrm{CMK}-3, \mathrm{Pd}_{\mathrm{IMP}} / \mathrm{CMK}-3$ and PdPVA$/ \mathrm{CMK}-3$ catalysts are $51 \%, 76 \%$ and $68 \%$. The Pd nanoparticle size of all three catalysts is similar, around $2.5 \mathrm{~nm}$ as measured from STEM images. The process for distinguishing Pd nanoparticles on the surface and inside pores from the reconstruction 3D data is illustrated in Fig.1. Fig.2a shows a representative 3D visualization of a segmented $\mathrm{Pd}_{\mathrm{IMP}} / \mathrm{CMK}-3$ catalyst with surface particles marked in green and particles inside the support marked in red. The relative amount of particles on the surface of each Pd/CMK-3 catalyst is calculated from several tomograms for each sample after segmentation and identification of the particles. The results are summarized in Fig.2b. The percentage of particles on the support surface is around $20 \%-30 \%$ in case of $\mathrm{Pd}_{\mathrm{IW}} / \mathrm{CMK}-3$, almost independent of the size of the CMK-3 support, whereas all $\mathrm{Pd}$ nanoparticles are locate on the CMK-3 surface in case of $\mathrm{Pd}_{\mathrm{PVA}} / \mathrm{CMK}-3$. In case of $\mathrm{Pd}_{\text {IMP }} / \mathrm{CMK}-3$, the amount of catalyst on the surface roughly scales with the surface area/volume ratio of the CMK-3 support, meaning that especially the smaller the CMK-3 exhibit a high Pd particle fraction on the surface. With the higher fraction of particle at the surface, $\mathrm{Pd}_{\mathrm{IMP}} / \mathrm{CMK}-3$ shows a higher activity compared to $\mathrm{Pd}_{\mathrm{IW}} / \mathrm{CMK}-3$, for which the accessibility of reactants to the catalyst inside particles is confined by the CMK-3 pore structure. The lower activity of $\mathrm{Pd}_{\mathrm{PVA}} / \mathrm{CMK}-3$ is possibly due to residual PVA stabilizing agent on the Pd nanoparticles, which might block some active sites. Fig.2c shows the 
size of the Pd particles on the surface and inside the CMK-3 support before and after catalysis as measured by electron tomography. While the size of the $\mathrm{Pd}$ nanoparticles in $\mathrm{Pd} \mathrm{d}_{\mathrm{IW}} / \mathrm{CMK}-3$ and $\mathrm{Pd}_{\mathrm{PVA}} / \mathrm{CMK}-3$ are almost unchanged, the Pd nanoparticles within and on the surface of $\mathrm{Pd} \mathrm{IMP}_{\mathrm{ICMK}} / \mathrm{CM}$ grew suggesting a poor stability of this catalyst despite the higher initial activity.

In this work, Pd nanoparticle locations and their size are precisely determined by quantitative electron tomography for different Pd/CMK-3 catalysts. The Pd nanoparticle location is one of the key factors for the activity, which is related to the confinement effects of the pore structure for active particles. Further work, evaluating e.g. particle shape/faceting and blocking of active sites will be performed in order to better understand the activity of the catalysts [3].

\section{References:}

[1] Aricò A S et al, Nature Materials 4(5) (2005), p. 366.

[2] Friedrich H et al, Journal of the American Chemical Society 129(33) (2007), p. 10249.

[3] Acknowledgment: Wu Wang is grateful for the financial support through the China Scholarship Council (CSC) for $\mathrm{PhD}$ research.
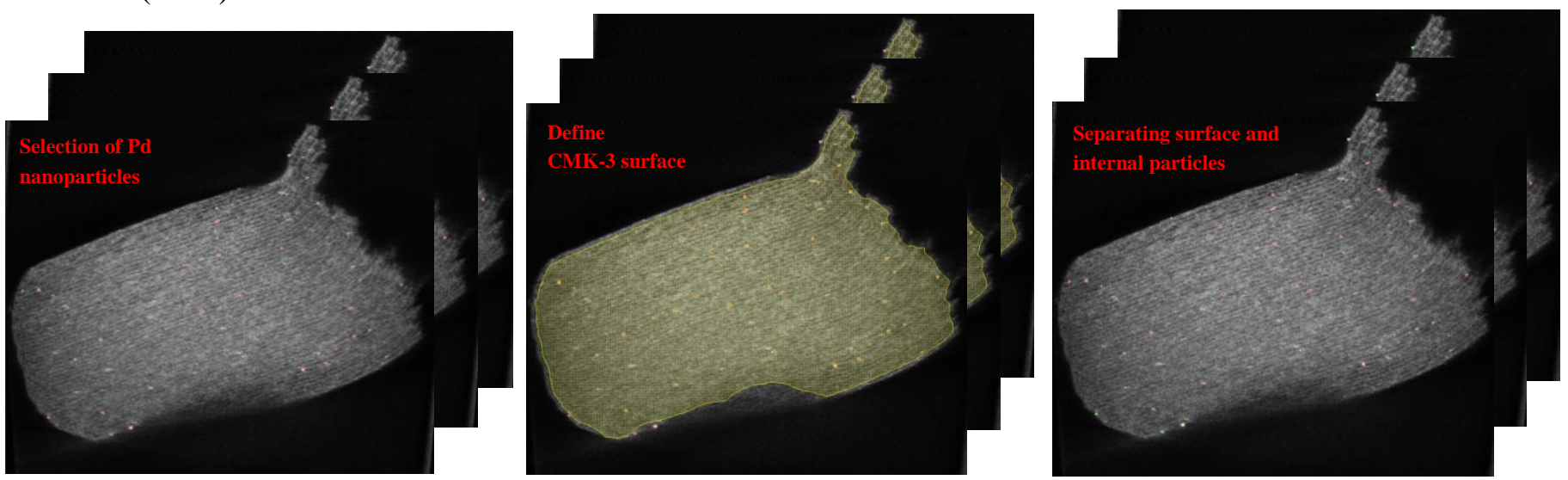

Figure 1. Particle segmentation and determination of particles on the surface and inside the CMK-3 support from the reconstruction 3D data.
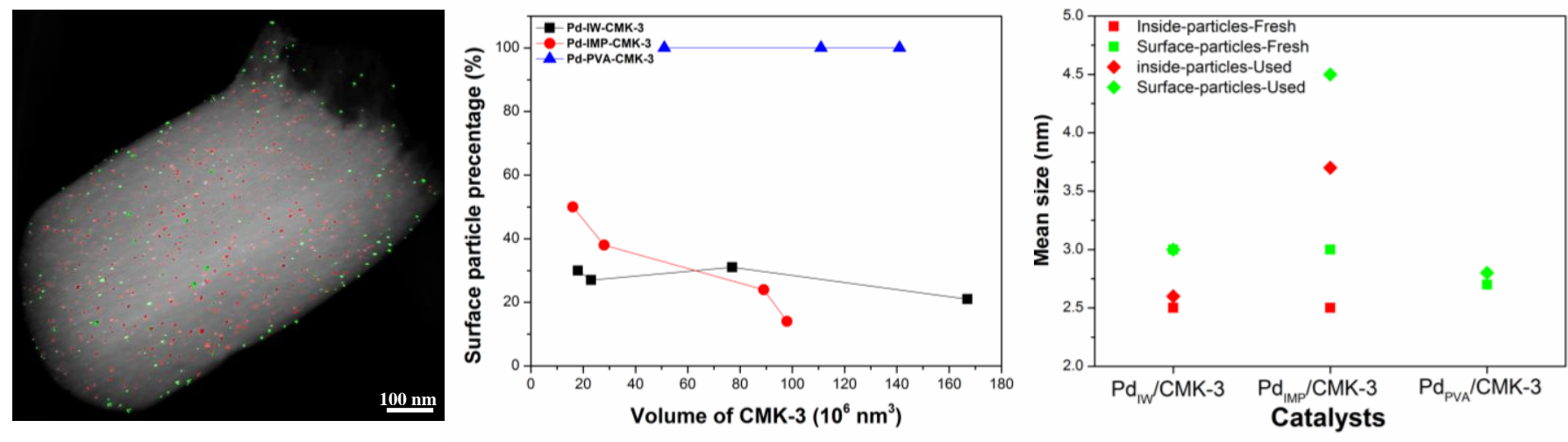

Figure 2. a) Representative $3 \mathrm{D}$ visualization of a $\mathrm{Pd}_{\mathrm{IMP}} / \mathrm{CMK}-3$ catalyst, b) fraction of particles on the surface for the as-prepared Pd/CMK-3 catalysts and c) mean particle diameter of Pd/CMK-3 before and after catalytic reaction. 